\title{
BMJ Open What is the relationship between renal function and visit-to-visit blood pressure variability in primary care? Retrospective cohort study from routinely collected healthcare data
}

\author{
Daniel S Lasserson, ${ }^{1,2}$ Nynke Scherpbier de Haan, ${ }^{3}$ Wim de Grauw, ${ }^{3}$ \\ Mark van der Wel, ${ }^{3}$ Jack F Wetzels, ${ }^{4}$ Christopher A O'Callaghan ${ }^{1}$
}

To cite: Lasserson DS, Scherpbier de Haan N, de Grauw W, et al. What is the relationship between renal function and visit-to-visit blood pressure variability in primary care? Retrospective cohort study from routinely collected healthcare data. BMJ Open 2016;6:e010702. doi:10.1136/bmjopen-2015010702

- Prepublication history and additional material is available. To view please visit the journal (http://dx.doi.org/ 10.1136/bmjopen-2015010702).

Received 27 November 2015 Revised 19 April 2016 Accepted 29 April 2016

CrossMark

For numbered affiliations see end of article.

\section{Correspondence to} Professor Christopher O'Callaghan; chris. ocallaghan@ndm.ox.ac.uk

\section{ABSTRACT}

Objective: To determine the relationship between renal function and visit-to-visit blood pressure (BP) variability in a cohort of primary care patients.

Design: Retrospective cohort study from routinely collected healthcare data.

Setting: Primary care in Nijmegen, the Netherlands, from 2007 to 2012.

Participants: 19175 patients who had a measure of renal function, and 7 separate visits with $\mathrm{BP}$ readings in the primary care record.

Outcome measures: Visit-to-visit variability in systolic BP, calculated from the first 7 office measurements, including SD, successive variation, absolute real variation and metrics of variability shown to be independent of mean. Multiple linear regression was used to analyse the influence of estimated glomerular filtration rate (eGFR) on BP variability measures with adjustment for age, sex, diabetes, mean $\mathrm{BP}$, proteinuria, cardiovascular disease, time interval between measures and antihypertensive use.

Results: In the patient cohort, $57 \%$ were women, mean (SD) age was 65.5 (12.3) years, mean (SD) eGFR was $75.6(18.0) \mathrm{mL} / \mathrm{min} / 1.73 \mathrm{~m}^{2}$ and SD systolic BP $148.3(21.4) \mathrm{mm} \mathrm{Hg}$. All BP variability measures were negatively correlated with eGFR and positively correlated with age. However, multiple linear regressions demonstrated consistent, small magnitude negative relationships between eGFR and all measures of BP variability adjusting for confounding variables.

Conclusions: Worsening renal function is associated with small increases in measures of visit-to-visit BP variability after adjustment for confounding factors.

This is seen across the spectrum of renal function in the population, and provides a mechanism whereby chronic kidney disease may raise the risk of cardiovascular events.

\section{INTRODUCTION}

Hypertension is a major vascular risk factor, and average blood pressure (BP) correlates

\section{Strengths and limitations of this study}

- We studied blood pressure (BP) variability in a patient cohort generalisable to primary care where the majority of patients with chronic kidney disease are diagnosed and monitored, as previously BP variability in chronic kidney disease (CKD) has only been studied in patients with advanced renal disease under specialist care.

- We examined measures of BP variability that are independent of mean BP, which have been shown to have greater prognostic significance than mean BP alone.

- We were not able to assess the effect of different exposures to antihypertensive medication.

- We did not have access to data on cardiovascular outcomes to test the clinical significance of visit-to-visit variability in this patient cohort.

with cardiovascular disease and death. ${ }^{1}$ Recently, visit-to-visit variability in BP measurements has been shown to convey an additional independent risk for cardiovascular events and to be associated with all-cause mortality in the general population. ${ }^{2}{ }^{3} \mathrm{BP}$ variability is reproducible within individuals over time in clinical trials ${ }^{4}$ and in observational data from routine care. ${ }^{4}$ The cardiovascular prognostic significance of $\mathrm{BP}$ variability is increasingly recognised, and the variability is not explained by poor medication adherence. ${ }^{5}$

Increased BP variability has been observed in small groups of patients with relatively advanced renal disease under specialist hospital care. In a small hospital cohort, visit-to-visit $\mathrm{BP}$ variability was independently associated with albuminuria and with increased renal vascular resistance as assessed by Doppler ultrasound. ${ }^{6}$ A study of 56 patients with non-diabetic chronic kidney disease (CKD) indicated a correlation between 
indices of BP variability and the rate of decline in renal function. ${ }^{7}$ In type 2 diabetes, visit-to-visit variability in systolic BP correlates with the level of albuminuria, ankle brachial pressure index ${ }^{8}$ and the probability of developing new albuminuria. ${ }^{9}$ In a set of secondary care patients with substantial renal impairment, there was a correlation between BP variability and a composite outcome of death and cardiovascular events. ${ }^{10}$ In patients on haemodialysis, visit-to-visit variability in $\mathrm{BP}$ is an independent predictor of vascular events, but this is complicated by the dependence of BP in this context on intradialytic fluid gain. ${ }^{11}$

However, as BP variability increases with mean BP, and renal disease is associated with increased mean $\mathrm{BP}$, the relationship between BP variability and renal impairment can only be established using metrics of BP variability that have been shown to be independent of mean BP. $^{12}$ None of the renal studies mentioned above reported statistics of BP variability that were demonstrated to be independent of the mean BP and, therefore, an evidence gap remains concerning whether declining renal function is associated with BP variability.

It is important to establish whether there is a real relationship between renal impairment and increased BP variability because the link might be causal and have important implications for therapy in CKD. Analysis of large sets of routinely collected healthcare data has demonstrated that CKD is an important independent risk factor for cardiovascular disease even after controlling for other known risk factors. ${ }^{13} 14$ The prevalence of CKD is around $5-10 \%$, and the majority of patients with CKD are managed by non-specialists or primary care physicians. ${ }^{15}{ }^{16}$ Given that a substantial proportion of the population have early stage CKD, it is therefore crucial to establish whether early stage CKD is associated with increased BP variability, as there could be major implications for population cardiovascular risk factor monitoring and treatment.

We therefore sought to determine whether renal function was associated with visit-to-visit $\mathrm{BP}$ variability using a large dataset of routinely collected healthcare data.

\section{METHODS}

We retrospectively defined a patient cohort (age $\geq 18$ years) using routinely collected healthcare data from 47 primary care practices in the Nijmegen region, the Netherlands, with a combined registered population of 207468 people, as part of the CONTACT study (Consultation of Nephrology by Telenephrology Allows optimal Chronic kidney disease Treatment in primary care), Netherlands Trial Registration code 2368, with approval from the Medical Research Ethics Committee Arnhem/Nijmegen, registration number 2010/187.

Data included demographic details, medical history with conditions diagnosed in the course of routine clinical practice, BP measurements, antihypertensive prescribing and renal function between 1 January 2008 and 1 January 2011. We did not seek to include patients receiving dialysis in this study population. The Chronic Kidney Disease Epidemiology Collaboration (CKD-EPI) formula was used to calculate estimated glomerular filtration rates (eGFRs) using the first creatinine measurements in the time period, which were either standardised to isotope dilution-mass spectrometry (IDMS), or subject to the appropriate correction factor for laboratories using the Jaffé technique. ${ }^{17}$ CKD-EPI eGFRs have been shown to correlate better with measured GFRs than eGFRs obtained with the Modification of Diet in Renal Disease (MDRD) study formula. ${ }^{18}$ The first seven office BP measurements were used to calculate variability metrics, as this approach has been shown to optimise reproducibility. ${ }^{12}$ Dutch primary care physicians and practice nurses have free access to the Dutch College of General Practitioners guideline on cardiovascular risk management for a clear description of standardised office BP measurement, which is in agreement with international standards.

Given that blood pressure variability is associated with mean BP, we calculated BP variability independent of mean (VIM) BP using standard formulae for measures that have prognostic significance for cardiovascular events. ${ }^{12}$ For the systolic and diastolic BP of individuals with at least seven BP measures, we calculated the SD, successive variation $(\mathrm{SV})$, average real variability (ARV), and transformed these to be independent of mean $\mathrm{BP}^{12}$ (see online supplementary information for formulae). Observed associations between renal function and BP variability (systolic BP metrics described above and also including maximum systolic BP), may be confounded by other predictors of variability, and we therefore tested the association between renal function and measures of BP variability using multiple stepwise linear regression adjusting for age, sex, mean BP, diabetes, history of cardiovascular disease (including vascular events, arrhythmia and heart failure), proteinuria, time interval between BP measures and class of antihypertensive drug prescribed. We included mean BP as a covariate to ensure that the effect of mean BP was truly adjusted for in regressions testing the relationship between VIM statistics and eGFR. Analyses were carried out using R (V.3.0.1) and SPSS V.21.

\section{RESULTS}

Among the 207468 total registered population, 162562 were over the age of 18 years, of whom 63073 (39\%) had renal function measured during the study period. Of these, $19175(30 \%)$ had at least seven BP measurements recorded at different visits. Table 1 lists the features of the group which had a mean age of 65.5 years, a mean blood creatinine concentration of $85.3 \mu \mathrm{mol} / \mathrm{L}$ $(0.97 \mathrm{mg} / \mathrm{dL})$, a mean eGFR of $75.6 \mathrm{~mL} / \mathrm{min} / 1.73 \mathrm{~m}^{2}$ and a mean systolic BP of $148.3 \mathrm{~mm} \mathrm{Hg}$.

The mean time interval from the first to the final seventh BP measurement was 22 months (SD 11 months). Mean systolic $B P$ rose with age $(R=0.19$, $\mathrm{p}<0.001)$ and with falling eGFR $(\mathrm{R}=-0.11, \mathrm{p}<0.001)$. Correlation analysis of transformed BP variability 
Table 1 Demographics of population studied $(n=19175)$

\begin{tabular}{ll}
\hline Age & $\mathbf{6 5 . 5}(\mathbf{1 2 . 3})$ years \\
\hline \% Female & $57 \%$ \\
CKD-EPI eGFR & $75.6(18.0) \mathrm{mL} / \mathrm{min} /$ \\
& $1.73 \mathrm{~m}^{2}$ \\
Creatinine & $85.3(23.0) \mathrm{\mu mol} / \mathrm{L}$ or \\
& $0.97(0.26) \mathrm{mg} / \mathrm{dL}$ \\
Diagnosed with diabetes & $37.5 \%$ \\
Number of antihypertensive drugs & $0-16.5$ \\
prescribed (\%, proportion of & $1-26.7$ \\
patients) & $2-30.1$ \\
& $3-18.8$ \\
& $4-6.8$ \\
& $5-1.0$ \\
& $6-0.1$ \\
Interval between 1st and 7th BP & $627(295)$ days \\
measure & $148.3(21.4) \mathrm{mm} \mathrm{Hg}$ \\
Systolic BP at baseline & \\
\hline Continuous data are presented as mean (SD). \\
BP, blood pressure; CKD-EPI, Chronic Kidney Disease \\
Epidemiology Collaboration formula; eGFR, estimated glomerular \\
filtration rate.
\end{tabular}

measures was consistent with their independence from mean BP ( $\mathrm{R}$ values; SD independent of mean (SDIM) $-0.009, p=0.23$; SV independent of mean (SVIM) -0.009 , $\mathrm{p}=0.22$; ARV independent of mean (ARVIM) -0.01, $\mathrm{p}=0.15$ ). Online supplementary table $\mathrm{S} 1$ shows the results of curve fitting to generate the transformed variables.

Table 2 shows the correlations between mean systolic $\mathrm{BP}$ and its variability with deteriorating eGFR and with increasing age. All measures of intraindividual BP variability increase progressively with declining eGFR and with increasing age.

The relationships of eGFR and age with SDIM, as an exemplar of the associations with VIM, are shown in figure $1 \mathrm{~A}, \mathrm{~B}$.

Multiple stepwise regression analysis was performed to examine the association between renal function and BP

Table 2 Correlation of systolic BP metrics with eGFR and with age

\begin{tabular}{|c|c|c|}
\hline Systolic BP metric & $\begin{array}{l}\text { Correlation } \\
\text { with eGFR }\end{array}$ & $\begin{array}{l}\text { Correlation } \\
\text { with age }\end{array}$ \\
\hline Mean & $-0.110^{* * *}$ & $0.190^{\star \star *}$ \\
\hline Maximum & $-0.121^{\star \star *}$ & $0.178^{\star \star \star}$ \\
\hline SD & $-0.116^{\star \star \star}$ & $0.115^{\star \star \star}$ \\
\hline SV & $-0.115^{\star \star \star}$ & $0.131^{\star \star \star}$ \\
\hline ARV & $-0.113^{\star \star \star}$ & $0.129^{\star \star \star}$ \\
\hline SDIM & $-0.086^{\star \star \star}$ & $0.054^{\star \star \star}$ \\
\hline SVIM BP & $-0.088^{\star \star \star}$ & $0.076^{\star \star \star}$ \\
\hline ARVIM BP & $-0.086^{\star \star \star}$ & $0.075^{\star \star \star}$ \\
\hline \multicolumn{3}{|c|}{$\begin{array}{l}{ }^{* * *} \mathrm{p}<0.001 \text {. } \\
\text { ARV, average real variability; ARVIM, ARV independent of mean; } \\
\text { BP, blood pressure; eGFR, estimated glomerular filtration rate; } \\
\text { SDIM, SD independent of mean; SV, successive variation; SVIM, } \\
\text { SV independent of mean. }\end{array}$} \\
\hline
\end{tabular}

variability. Table 3 shows the standardised $\beta$ coefficients and associated $p$ values from multiple regression analyses of eGFR on BP variability metrics using, age, sex, mean systolic BP, diabetes, cardiovascular disease, proteinuria, interval between BP measures and the class of antihypertensive drug prescribed as covariates (medication classes are not shown, see online supplementary table S2). Non-standardised coefficients for categorical variables are shown in online supplementary table S3).

Lower eGFR was consistently associated with increasing measures of BP variability (both with and without transformation to be independent of mean BP), as evidenced by the significant negative standardised $\beta$ coefficients across the regressions in the presence of potential confounders. Although the presence of proteinuria was not associated with any measure of BP variability, a history of cardiovascular disease was positively associated with increased BP variability. All classes of antihypertensive medication were positively associated with measures of BP variability where significant relationships were observed (see online supplementary table S2).

Greater BP variability was associated with longer time intervals between BP measures, as evidenced by the positive coefficients where significant relationships were observed.

\section{DISCUSSION}

Both mean BP and visit-to-visit BP variability increased with age and with worsening renal function in this large community-based population. Further analysis with multiple linear regression demonstrated that worsening renal function remains significantly associated with increased BPVIM, even after adjustment for age, sex, diabetes, history of cardiovascular disease, mean systolic blood pressure, interval between $\mathrm{BP}$ measures, proteinuria and the class of antihypertensive drug prescribed. This is important because visit-to-visit BP variability over the medium term is a strong predictor of cardiovascular outcome independently of mean $\mathrm{BP}^{3}{ }^{3}$ In addition, this relationship between renal function and $\mathrm{BP}$ variability may explain, in part, the association between renal function and cardiovascular disease, which remains even after controlling for other established cardiovascular risk factors. ${ }^{15}$

Hitherto, the relationship between renal disease and BP variability has not been studied in large unselected community-based populations. In specialist services which typically care for patients with advanced renal disease, or those requiring renal replacement therapy, visit-to-visit variability in $\mathrm{BP}$ has been linked to poorer renal $^{6}$ and cardiovascular outcomes. ${ }^{19}$ In settings, such as the UK or the Netherlands, where guidelines exist for specialist referral, ${ }^{20}{ }^{21}$ there is substantial enrichment in specialist service populations for advanced CKD stage, rapidly declining renal function, or significant proteinuria. Observational studies of these groups for prognostic factors has an uncertain generalisability to the much 

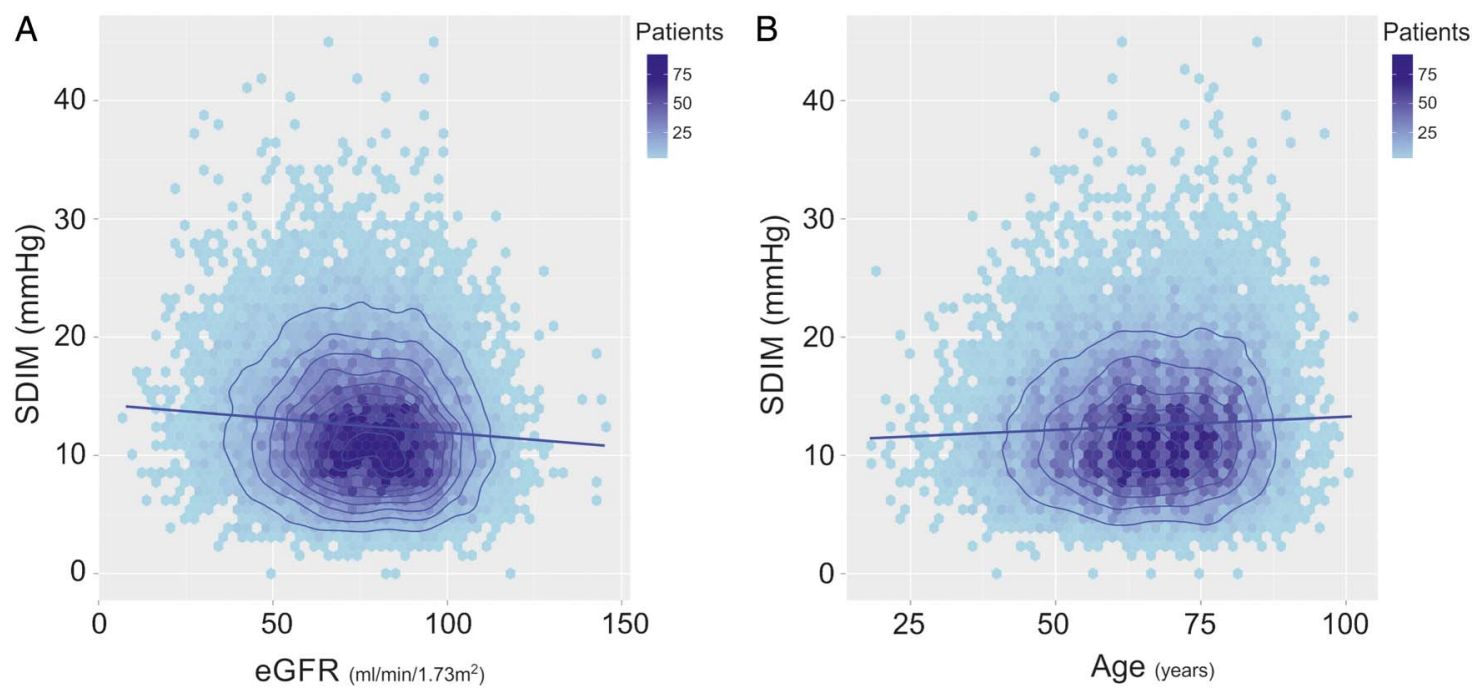

Figure 1 Renal function and age, both associated with blood pressure variability. Colours indicate the number of data points in each shaded area of the plot. The number of patients represented by each coloured polygon, that is, the data frequency, is indicated by the colour scale. Contours are added to aid visualisation of the shape of the frequency distribution across the two-dimensional plot. The contours are derived from kernel density estimation of the data frequency, and represent the boundaries of intervals of equal magnitude in the data frequency. The regression line is shown. (A) The relationship between SD independent of mean (SDIM) and estimated glomerular filtration rate (eGFR). (B) The relationship between SDIM and age.

larger, but less severely affected, population monitored predominantly by non-specialists or primary care physicians. Furthermore, none of the previous studies of renal disease have used metrics of $\mathrm{BP}$ variability that have been shown to be independent of mean BP, and so, do not clearly distinguish between the effects of raised systolic $\mathrm{BP}$ and $\mathrm{BP}$ variability.

The results of the regression analyses showed a consistent positive relationship between the presence of cardiovascular disease and $\mathrm{BP}$ variability, a finding that is consistent with the known prognostic significance of BP variability. ${ }^{22}$ Importantly, the time interval between BP measures was positively associated with BP variability, indicating that longer durations between measures were associated with greater variability. This counters the hypothesis that associations between greater BP variability and reduced renal function are an artefact of acute illness where clinical monitoring will be more frequent, or due to increased monitoring at the time of initiation of antihypertensive drugs. If acute illness or the initiation of antihypertensive therapy was responsible for increasing BP variability, one would expect greater variability to be associated with shorter time intervals between $\mathrm{BP}$ measures in a period of clinical instability or medication change. We presented standardised coefficients for all included variables in order to facilitate a clear clinical interpretation of the relative contributions of the predictors of the $\mathrm{BP}$ variability measures.

A limited post hoc analysis of around 2000 patients selected for the ASCOT-BPLA trial showed a weak relationship between blood creatinine and BP variability, but the trial design undermines the generalisability of this analysis to representative patient populations. ${ }^{22}$ The

Table 3 Standardised $\beta$ coefficients and associated $p$ values from multiple linear regressions of eGFR on measures of BP variability adjusted for potential confounders (medication classes not shown)

\begin{tabular}{|c|c|c|c|c|c|c|c|}
\hline $\begin{array}{l}\text { Measure of BP } \\
\text { Variability } \\
\end{array}$ & $\begin{array}{l}\text { eGFR } \\
\text { Std } \beta\end{array}$ & $\begin{array}{l}\text { Age } \\
\text { Std } \beta\end{array}$ & $\begin{array}{l}\text { Sex } \\
\text { Std } \beta \\
\end{array}$ & $\begin{array}{l}\text { Mean BP } \\
\text { Std } \beta\end{array}$ & $\begin{array}{l}\text { Vascular disease } \\
\text { Std } \beta\end{array}$ & $\begin{array}{l}\text { BP interval } \\
\text { Std } \beta\end{array}$ & $\begin{array}{l}\text { Diabetes } \\
\text { Std } \beta \\
\end{array}$ \\
\hline SD & $-0.04^{\star \star \star}$ & - & $0.06^{\star * \star}$ & $0.37^{\star \star \star}$ & $0.07^{\star \star \star}$ & - & $-0.06^{\star \star \star}$ \\
\hline ARV & $-0.04^{\star \star \star}$ & $0.02^{* \star *}$ & $0.06^{\star * *}$ & $0.31^{\star \star \star}$ & $0.06^{\star \star \star}$ & $0.02^{*}$ & $-0.035^{\star \star \star}$ \\
\hline SRV & $-0.04^{\star \star *}$ & $0.02^{*}$ & $0.07^{\star * *}$ & $0.32^{\star \star \star}$ & $0.06^{* * *}$ & $0.02^{*}$ & $-0.03^{\star \star *}$ \\
\hline Max systolic & $-0.02^{\star \star \star}$ & $-0.01^{*}$ & $0.03^{\star \star \star}$ & $0.88^{\star \star \star}$ & $0.03^{\star \star \star}$ & - & $-0.03^{\star \star \star}$ \\
\hline SDIM & $-0.05^{\star \star *}$ & - & $0.07^{\star \star \star}$ & $-0.04^{\star \star \star}$ & $0.08^{\star \star \star}$ & - & $-0.07^{\star \star \star}$ \\
\hline ARVIM & $-0.04^{\star \star \star}$ & $0.03^{*}$ & $0.06^{\star \star \star}$ & $-0.04^{\star \star \star}$ & $0.07^{\star \star \star}$ & $0.02^{*}$ & $-0.04^{\star \star \star}$ \\
\hline SRVIM & $-0.05^{\star \star \star}$ & $0.03^{\star *}$ & $0.07^{\star \star \star}$ & $-0.04^{\star \star \star}$ & $0.07^{\star \star \star}$ & $0.02^{*}$ & $-0.041^{\star \star \star}$ \\
\hline
\end{tabular}

${ }^{*} \mathrm{p}<0.05,{ }^{* *} \mathrm{p}<0.01,{ }^{* * *} \mathrm{p}<0.001$

ARV, average real variability; ARVIM, ARV independent of mean; BP, blood pressure; eGFR, estimated glomerular filtration rate; SDIM, SD independent of mean; SRV, successive real variability; SRVIM, SRV independent of mean.

Coefficients shown if included in stepwise multiple regression. 
study excluded anyone with a creatinine of $>200 \mu \mathrm{mol} / \mathrm{L}$ $(2.26 \mathrm{mg} / \mathrm{dL})$, with clinically important renal disease, with secondary hypertension (which could include renal disease), or any concomitant disease requiring calcium channel blockers, angiotensin converting enzyme inhibitors, $\beta$ blockers, or diuretics. These criteria would likely exclude the majority of patients with chronic kidney disease. Further, the study excluded anyone $<55$ years of age, eGFRs were not reported, and it is unclear whether creatinine assays were standardised to IDMS values. Creatinine is influenced by both age and renal function-in part because of age-related changes in muscle mass-and the analysis was weakened in this older trial population by the comparison of creatinine values across different ages.

The relationship we observed between CKD and BP variability in a wider population demonstrates that, even in the early stages of renal dysfunction, BP variability is present at a level associated with significant cardiovascular risk. The measures of variability (ARV, SV and those transformed to be independent of mean) in eGFR ranges corresponding to CKD stage 4 in this study are similar to those seen in cohorts of patients with transient ischaemic attack (TIA) in the UK-TIA trial and the European Carotid Surgery Trial. ${ }^{12}$ Furthermore, the timeframe of BP variability measurement in this study (mean time between BP measures of 104 days) is consistent with the time frames between repeat BP measures during follow-up in the trials establishing increased BP variability and its prognostic significance. ${ }^{12} 22$

Our study has some limitations. The population studied exists in a healthcare context where the value of treating hypertension is recognised, and this is likely to reduce the slope of the relationship between mean systolic BP and eGFR. In addition, the variability associated with the estimation of renal function, ${ }^{16}$ and intrinsic error will result in regression dilution bias. However, this will bias the study towards the null hypothesis, such that the strength of the relationship between renal function and $\mathrm{BP}$ variability will be underestimated rather than overestimated. Error associated with the measurement of $\mathrm{BP}$ variability will widen the confidence limits associated with the relationship. Furthermore, while our adjustment for antihypertensive use included drug-class specific effects, we were not able to assess the effect of exposure by examining drop-in and drop-out of treatment, but given that variability may be affected by some drugs more than others, ${ }^{23}$ this constraint is also likely to reduce rather than enhance the associations that we have found. The findings are limited to patients with multiple BP readings taken at their general practice, and at least one measure of renal function. Nevertheless, despite these limitations, we have found consistent and significant associations between measures of renal function and $\mathrm{BP}$ variability.

Our results demonstrate that the relationship between eGFR and BP variability exists across the spectrum of eGFR which corresponds to different stages of CKD.
This relationship is consistent with a causative relationship between diminished eGFR and BP variability, but causation cannot be established in an observational study. Nevertheless, this may prove to have clinical usefulness in determining vascular risk and prognosis, as we have demonstrated that BP variability is readily detectable and quantifiable in retrospective data in the nonspecialist setting.

Studies of the prognostic significance of BP variability in general CKD populations are required as monitoring $\mathrm{BP}$ variability may refine the prediction of cardiovascular risk. The correlations that we have identified are of small magnitude, but they are independent, and given the population prevalence of CKD, this correlation will have consequences for cardiovascular risk at the population level. Clinical trials are needed in CKD to establish the value or otherwise for renal and cardiovascular outcomes of tailoring antihypertensive therapy to minimise $\mathrm{BP}$ variability.

\section{Author affiliations}

${ }^{1}$ Nuffield Department of Medicine, University of Oxford, Oxford, UK

${ }^{2}$ Department of Geratology, NIHR Oxford Biomedical Research Centre, Oxford University Hospitals NHS Trust, Oxford, UK

${ }^{3}$ Department of Primary and Community Care, Radboud University Nijmegen Medical Centre, Nijmegen, The Netherlands

${ }^{4}$ Department of Nephrology, Radboud University Nijmegen Medical Centre, Nijmegen, The Netherlands

Contributors DSL and COC conceived the idea, designed the analysis, undertook the analysis and wrote the manuscript. NSdH, WJdG, MvdW and JW interpreted the data and wrote the manuscript.

Funding The Dutch Kidney Foundation. DSL is supported by the NIHR Oxford Biomedical Research Centre. The views expressed are those of the authors and not necessarily those of the NIHR, the NHS, or the Department of Health.

Competing interests None declared.

Ethics approval Medical Research Ethics Committee Arnhem/Nijmegen registration number 2010/187.

Provenance and peer review Not commissioned; externally peer reviewed.

Data sharing statement No additional data are available.

Open Access This is an Open Access article distributed in accordance with the terms of the Creative Commons Attribution (CC BY 4.0) license, which permits others to distribute, remix, adapt and build upon this work, for commercial use, provided the original work is properly cited. See: http:// creativecommons.org/licenses/by/4.0/

\section{REFERENCES}

1. MacMahon S, Peto R, Cutler J, et al. Blood pressure, stroke, and coronary heart disease. Part 1, Prolonged differences in blood pressure: prospective observational studies corrected for the regression dilution bias. Lancet 1990;335:765-74.

2. Muntner $P$, Shimbo D, Tonelli $M$, et al. The relationship between visit-to-visit variability in systolic blood pressure and all-cause mortality in the general population: findings from NHANES III, 1988 to 1994. Hypertension 2011;57:160-6.

3. Rothwell PM. Limitations of the usual blood-pressure hypothesis and importance of variability, instability, and episodic hypertension. Lancet 2010;375:938-48.

4. Muntner P, Joyce C, Levitan EB, et al. Reproducibility of visit-to-visit variability of blood pressure measured as part of routine clinical care. J Hypertens 2011;29:2332-8.

5. Muntner P, Levitan EB, Joyce C, et al. Association between antihypertensive medication adherence and visit-to-visit variability of blood pressure. J ClinHypertens (Greenwich) 2013;15:112-17. 
6. Kawai T, Ohishi M, Kamide K, et al. The impact of visit-to-visit variability in blood pressure on renal function. Hypertens Res 2012;35:239-43.

7. Yokota K, Fukuda M, Matsui Y, et al. Impact of visit-to-visit variability of blood pressure on deterioration of renal function in patients with non-diabetic chronic kidney disease. Hypertens Res 2013;36:151-7.

8. Okada H, Fukui M, Tanaka M, et al. Visit-to-visit variability in systolic blood pressure is correlated with diabetic nephropathy and atherosclerosis in patients with type 2 diabetes. Atherosclerosis 2012;220:155-9.

9. Okada H, Fukui M, Tanaka M, et al. Visit-to-visit blood pressure variability is a novel risk factor for the development and progression of diabetic nephropathy in patients with type 2 diabetes. Diabetes Care 2013;36:1908-12.

10. Mallamaci F, Minutolo R, Leonardis D, et al. Long-term visit-to-visit office blood pressure variability increases the risk of adverse cardiovascular outcomes in patients with chronic kidney disease. Kidney Int 2013;84:381-9.

11. Rossignol $\mathrm{P}$, Cridlig J, Lehert $\mathrm{P}$, et al. Visit-to-visit blood pressure variability is a strong predictor of cardiovascular events in hemodialysis: insights from FOSIDIAL. Hypertension 2012;60:339-46.

12. Howard SC, Rothwell PM. Reproducibility of measures of visit-to-visit variability in blood pressure after transient ischaemic attack or minor stroke. Cerebrovasc Dis 2009;28:331-40.

13. Go AS, Chertow GM, Fan D, et al. Chronic kidney disease and the risks of death, cardiovascular events, and hospitalization. $N$ Engl J Med 2004;351:1296-305.

14. Tonelli M, Muntner P, Lloyd A, et al. Risk of coronary events in people with chronic kidney disease compared with those with diabetes: a population-level cohort study. Lancet 2012;380:807-14.

15. Coresh J, Stevens LA, Levey AS. Chronic kidney disease is common: what do we do next? Nephrol Dial Transplant 2008;23:1122-5.

16. O'Callaghan CA, Shine B, Lasserson DS. Chronic kidney disease: a large-scale population-based study of the effects of introducing the CKD-EPI formula for eGFR reporting. BMJ Open 2011;1: e000308.

17. Levey AS, Coresh J, Greene T, et al. Expressing the modification of diet in renal disease study equation for estimating glomerular filtration rate with standardized serum creatinine values. Clin Chem 2007:53:766-72.

18. Levey AS, Stevens LA, Schmid $\mathrm{CH}$, et al. A new equation to estimate glomerular filtration rate. Ann Intern Med 2009;150:604-12.

19. Di lorio B, Pota A, Sirico ML, et al. Blood pressure variability and outcomes in chronic kidney disease. Nephrol Dial Transplant 2012;27:4404-10.

20. National Coordinating Centre for Chronic Conditions. CHRONIC KIDNEY DISEASE. National clinical guideline for early identification and management in adults in primary and secondary care. London: Royal College of Physicians, 2008.

21. De Grauw WJC, Kaasjager HAH, Bilo HJG, et al. Landelijke Transmurale Afspraak Chronische nierschade. Huisarts Wet 2009;52:586-97.

22. Rothwell PM, Howard SC, Dolan E, et al. Prognostic significance of visit-to-visit variability, maximum systolic blood pressure, and episodic hypertension. Lancet 2010;375:895-905.

23. Webb AJ, Rothwell PM. Effect of dose and combination of antihypertensives on interindividual blood pressure variability: a systematic review. Stroke 2011;42:2860-5. 